\title{
Toward Automated Assessment of User Experience in Extended Reality
}

\author{
Saba Gholizadeh Ansari \\ dept. Information and Computing Sciences, Utrecht University, the Netherlands \\ s.gholizadehansari@uu.nl
}

\begin{abstract}
Designers of extended reality systems need to predict users feedback about designed elements to evaluate their systems. Manual user experience testing can not cover all preferences of users and user-system interactions. To improve and accelerate this process, automated user experience testing is a field of growing interest. Since users' emotions affect their experience, the automated testing framework should represent users with different emotional states. In this study, we propose an approach to deploy an automated user experience testing framework using BDI test agents which work with a computational model of emotion to regulate their testing behavior.

Keywords-Automated playtesting, Emotional user experience testing, Extended reality, Agent simulation
\end{abstract}

\section{INTRODUCTION}

Extended reality (XR) systems like rich 3D virtual worlds, virtual reality (VR), and Augmented Reality (AR) have been flourishing in various domains from entertainment to combat training and critical applications. With their increasing popularity, the demand for automated testing to assure functional as well as non-functional correctness grows. Despite some developments towards automated playtesting, toolsets mainly work on fixed simple test scenarios which furthermore are vulnerable to system changes during development. Another major problem in XR system development, one that our thesis will focus on, is to predict how the user would experience the XR system (user experience/UX) when exposed to it.

If exposed to contents and elements which do not fulfil their expectations, users will abandon the system. So, it is essential for XR designers to predict how users would interact with the system [1]. On one hand, aspects such as user preferences and functional interactions between the users and the system, and among themselves, need to be taken into account. On the other hand, from psychological perspective, some situations result in emotional states which affect the user experience. To meet this aim, designers might take various approaches, from simply imagining what different types of users might do, to analyzing data from manual test by human testers. While more precise, this unfortunately also requires excessive hours of testing while available resources are often limited. The testers, moreover, might not represent all user types and their emotional attitudes towards the XR system. The proposed approach will be integrated with a search-based testing to increase test coverage.

This is a part of IV4XR project Funded by EU, grant nr. 856716 The PhD thesis is advised by I. S. W. B. Prasetya, Utrecht University.
Thesis' goal. In order to assist XR designers to evaluate whether their implemented ideas provide the user experience they are designed for, my thesis goal is to develop automated UX testers which can represent users with different emotional preferences and relevant emotional states. An agent-based approach will be chosen, with which intelligent agents are deployed into the XR system to generate tests based on given UX test specifications. An intelligent agent is a computer program having the ability to provide autonomy, reactivity, sociability and pro-activeness to observe and act upon an environment and direct its activity towards achieving given goals [2]. Emotions also need to be considered, as they also regulate the behavior of humans e.g. in coping with difficulties. Without considering them the generated UX tests would not be human-like/realistic. This will be achieved by deploying a computational model of emotion [3], designed to provide affective processing in our intelligent test agents. Additionally, the combination of our proposed approach with search based testing mechanisms [4] will be investigated, to improve the confidence of UX testing and bring about higher coverage.

\section{RELATED WORK}

Different approaches, from heuristic to machine learning, have been adopted to introduce automation in UX testing, or to automate related tasks. E.g. in [5] a framework is proposed for estimating level difficulty of match-3 games in which the CNN-based agent is trained on human-player data. In [6] automated playtesting with personas is implemented using a variation of Monte-Carlo-Tree Search where Upper Confidence Bound is replaced by genetic programming. This generative model helps designers to evaluate playability for players with different preferences. Despite the potential benefit, combining emotional modelling and software engineering is still mostly unexplored. There were some attempts to identify the software engineering elements of emotional modeling to improve its development process [7], but the opposite direction, such as the use of computational model of emotion for automated UX testing, is not a topic that has been studied widely.

\section{EXPECTED CONTRIBUTIONS}

Objective-1: develop an automated approach to test the UX quality, e.g. how entertaining a new level is? The approach will be built on top a 'BDI' agent framework [2] which will be discussed later. BDI agents will be turned into test 
agents, and extended with a computational model of emotion [3], enabling them to test the level of UX characteristics. Furthermore, as remarked before, it is essential that the agents can closely simulate human playtesters. In other words, they should generate behavior which is human-like, which is highly challenging. This will be addressed as well.

Objective-2: identify which mental states are essential for $U X$ testing and an appropriate way to model them. This will be elaborated in methodology section.

Objective-3: to model testers' knowledge and skills relevant for generating human-like behavior into test agents. While these can be programmed as sets of rules, a more interesting option is to obtain them through a learning mechanism for the agents. To address it, however, we need to do further research.

Objective-4: increase the coverage of UX testing. After having the basic automated UX testing framework, improving the confidence of such testing will take the priority, so our framework needs to generate various explorations on e.g. the given new content, to make sure enough variations of possible user behavior are tested. At first, we need to provide a technical definition of UX coverage. Then, based on the definition, search-based testing algorithms [4] will be adopted to achieve a diversified coverage of the test goals.

\section{Methodology and Current Stage}

To develop our UX test agents, the Belief-Desire-Intention (BDI) model of agency [3] is selected. This model is a classic paradigm to formalize the internal architecture of software agents in agent-oriented systems. A BDI agent has some goals, also called desires, and sequences of actions called plans to achieve the goals and react to occurring events. When an agent has decided on pursuing a goal with a certain plan, it establishes an intention towards a sequence of plan actions. For our purpose, an agent will have testing goals.

Furthermore, computational models of emotion inspired by emotional theories aim to create emotionally intelligent agents being capable of expressing emotions in response to perceived events [8]. Emotions lead an agent to coping processes where the agent can selectively respond to feelings and include both problem-focused coping strategies, in which the agent takes actions in the world, and emotion-focused strategies.

A well known appraisal process in emotion used in many computational frameworks of emotion is inspired by the OCC model [8]. This model structures 22 emotion types (e.g. joy, satisfaction, hope, disappointment, distress and fear) based on focus of attention. All emotions are valenced reactions which can be directed by either consequence of events, consequence of agents' actions, or aspects of object. Different situations of XR system will be labeled emotionally by XR designers. The computational model of emotion will be implemented using these labels in the framework. Then the framework will be integrated with a proper search-based testing algorithm.

Since we will base our framework on BDI, we will use the OCC model of emotion which has been formalized using BDI logic [9]. At this stage, our team is finishing the development of the underlying BDI agent programming framework named
Aplib $^{1}$ [10] which offers a Domain Specific Language (DSL) to program agents, embedded in Java to access all benefits of Java programming language and interface to XR systems.

To make sure our results are deployable in a real industrial setup, the framework will to be tested in three different XR systems. These include advanced 3D games, where entertainment is an essential UX aspect, as well as a mission critical simulation where the UX of various test-scenarios needs to be verified if they fulfil the training purpose defined by the instructor. Fig. 1 shows our first case study which is a $3 \mathrm{D}$ game. Developers must verify whether all rooms needing specific switches, are reachable. The game contains many other interactive objects like hazardous dynamic entities which may cause an agent to fall into an inescapable stuck state whose presence must be balanced to keep the game interesting.

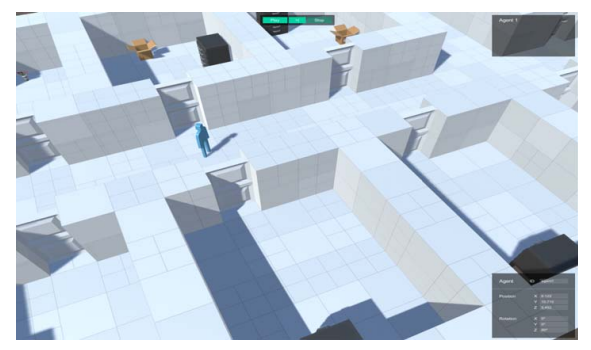

Fig. 1. An under development 3D game for automated XR testing framework

\section{Future Plan AND EXPECTATIONS}

Project plan. The $\mathrm{PhD}$ project will take four years. During the first year we will focus on Objective-2 and Objective-3, so we will develop our agent-based testing framework [10] with modeling the emotional states. This will be validated with a case study. In the second year, we will deploy our framework to support more emotions. Meanwhile, a technical definition for emotional coverage in UX will be given and an approach for increasing UX test coverage will be adopted. In the fourth year, we will address the challenge of multi-user setup which is essential for systems with multiple users as well as systems that simulate multiple humans. This will require us to model cooperative as well as competitive behavior in our framework, and also to understand and model how an agent can trigger a specific emotion on the other ones.

Validation. The proposed UX testing framework will be validated using a popular 3D game as well as a mission critical 3D simulator. Moreover, users' emotions affect facial expression, electroencephalogram and heart rate [11]. To assure the automated agents represent users properly, our results will be evaluated with real users experience and their detected emotions.

\section{ACKNOWLEDGMENT}

I would like to thank Frank Dignum and Mehdi Dastani for their advice. I also wish to thank Pedro M. Fernandes for his suggestions.

\footnotetext{
${ }^{1}$ https://iv4xr-project.github.io/aplib/
} 


\section{REFERENCES}

[1] A. Drachen and A. Canossa, "Towards gameplay analysis via gameplay metrics," in Proceedings of the 13th international MindTrek conference: Everyday life in the ubiquitous era, pp. 202-209, ACM, 2009.

[2] M. Wooldridge, "Intelligent agents," Multiagent systems, vol. 35, no. 4 p. 51, 1999.

[3] L.-F. Rodríguez and F. Ramos, "Development of computational models of emotions for autonomous agents: a review," Cognitive Computation, vol. 6, no. 3, pp. 351-375, 2014.

[4] P. McMinn, "Search-based software test data generation: a survey," Software testing, Verification and reliability, vol. 14, no. 2, pp. 105 $156,2004$.

[5] S. F. Gudmundsson, P. Eisen, E. Poromaa, A. Nodet, S. Purmonen, B. Kozakowski, R. Meurling, and L. Cao, "Human-like playtesting with deep learning," in 2018 IEEE Conference on Computational Intelligence and Games (CIG), pp. 1-8, IEEE, 2018.

[6] C. Holmgard, M. C. Green, A. Liapis, and J. Togelius, "Automated playtesting with procedural personas with evolved heuristics," IEEE Transactions on Games, 2018.

[7] E. Osuna, L.-F. Rodríguez, J. O. Gutierrez-Garcia, and L. A. Castro, "Development of computational models of emotions: A software engineering perspective," Cognitive Systems Research, vol. 60, pp. 1-19, 2020.

[8] A. Ortony, G. Clore, and A. Collins, "The cognitive structure of emotions. cam (bridge university press," Cambridge, England, 1988.

[9] C. Adam, A. Herzig, and D. Longin, "A logical formalization of the occ theory of emotions," Synthese, vol. 168, no. 2, pp. 201-248, 2009.

[10] I. Prasetya, "Aplib: Tactical programming of intelligent agents," arXiv preprint arXiv:1911.04710, 2019.

[11] A. Drachen, L. E. Nacke, G. Yannakakis, and A. L. Pedersen, "Correlation between heart rate, electrodermal activity and player experience in first-person shooter games," in Proceedings of the 5th ACM SIGGRAPH Symposium on Video Games, pp. 49-54, ACM, 2010. 